\title{
Ein Modell für die stationäre medizinische Versorgung von Menschen mit somatischen und psychischen Erkrankungen
}

\author{
Auf der Krankenstation werden Patienten behandelt, die unter mehreren Krank- \\ heiten gleichzeitig leiden. Im Vordergrund stehen Suchterkrankungen mit den \\ dazugehörigen Folgeerkrankungen. Zusätzlich leiden alle Patienten unter psych- \\ iatrischen Erkrankungen. Weil eine ambulante Pflege und Betreuung nach der \\ stationären Behandlung oft nicht gewährleistet ist, bleiben die Patienten immer \\ wieder für die Übergangspflege im Sune-Egge.
}

Ulrich Erlinger ${ }^{a}$, Robert Zink ${ }^{b}$, Daniela Dyntar

a Dr. med., M.P.H., Chefarzt Stadtärztlicher Dienst Zürich (bis 31.1.2012)

b Dr. med., Leitender Arzt Sune-Egge, Städtische Gesundheitsdienste Zürich

c Dr. sc. nat. ETH, wissenschaftliche Mitarbeiterin, Städtische Gesundheitsdienste Zürich

Zu diesem Thema siehe auch das Porträt über A. Seidenberg auf Seite 342.

\footnotetext{
* Die Literaturangaben finden sich unter ww.saez.ch $\rightarrow$ Aktuelle Nummer oder $\rightarrow$ Archiv $\rightarrow 2012 \rightarrow 9$
}

Korrespondenzadresse: Dr. med. Robert Zink Leitender Arzt

Krankenstation Sune-Egge Konradstrasse 62

CH-8005 Zürich

robert.zink[at]swsieber.ch
Die wesentliche Verbreitung der Drogen begann Ende der sechziger Jahre, der erste Drogentote in Zürich wurde 1972 erfasst [1-3]*. Im Zuge der steigenden Zahl von Drogentoten, der mit dem Drogenkonsum verbundenen Verwahrlosung und der Verbreitung von Aids setzte sich die Medizin mit den Besonderheiten der Behandlung von Drogenpatienten auseinander [4-7]. Der Umgang mit drogenabhängigen Menschen in der Schweiz, der sich nach einer wissenschaftlich evaluierten Experimentierphase etablieren konnte [8], fand und findet weltweit Beachtung und wurde oft kopiert, weil hier pragmatisch und effektiv ge- und behandelt wird [9-11], auch wenn bereits etablierte Behandlungsprogramme nicht mehr ausreichen $[8,10,12,13]$.

\section{Der Sune-Egge}

Durch das Elend der offenen Drogenszenen in Zürich im Jahre 1989 bewegt, gründete Pfarrer Ernst Sieber die sozialmedizinische Krankenstation SuneEgge, die sich heute zu einem Kompetenz-Zentrum zur Behandlung von Menschen mit Drogenproblemen entwickelt hat. Im Rahmen der unbürokratischen Soforthilfe bei medizinischen und sozialen Problemen behandelt und berät der Sune-Egge jährlich mehrere hundert Patienten in den folgenden Settings: Methadonabgabe im Ambulatorium, ärztliche Praxis, Pflegestation für Akutpatienten und eine Abteilung für Langzeitkranke, bei denen die Aidserkrankung fortgeschritten ist. Weil aufgrund mangelnder Integration der Patienten eine ambulante Pflege und Betreuung nach der Demissio aus der stationären Behandlung häufig nicht durch die Spitex oder Familienmitglieder gewährleistet ist, verweilen die Patienten immer wieder für die Übergangspflege im Sune-Egge. Es besteht eine enge Zusammenarbeit
Un modèle pour la prise en charge stationnaire de patients atteints de maladies somatiques et psychiques Fondée dans les années 80 dans le sillage des efforts déployés pour fermer la scène ouverte de la drogue à Zurich, la clinique Sune-Egge figure sur la liste cantonale 2012 des hôpitaux en tant qu'hôpital de soins aigus destiné aux personnes victimes d'addictions. Les patients pris en charge dans cette clinique souffrent de maladies multiples, principalement de maladies de l'addiction et de maladies secondaires associées. II s'agit d'une part de maladies infectieuses comme l'hépatite $B$ et $C$ et le VIH, et d'autre part de maladies de la peau liées à l'injection de drogues par voie intraveineuse (abcès, infections profondes de la peau et des os) ou encore de séquelles tardives comme la défaillance du foie et des reins. Parallèlement aux maladies de la dépendance et aux maladies somatiques, tous les patients souffrent également de maladies psychiques.

mit dem UniversitätsSpital Zürich und den übrigen Krankenhäusern, den Fachstellen der privaten und öffentlichen Sucht- und Drogenhilfe und des Gesundheitswesens. Besondere Bedeutung wird der 
Vernetzung mit den anderen Einrichtungen der Sozialwerke Pfarrer Sieber (SWS) sowie weiteren kirchlichen, privaten und staatlichen Institutionen beigemessen. Teil des interdisziplinären Behandlungsteams sind der ärztliche Leiter, ein Facharzt für Allgemeine Innere Medizin sowie ein Facharzt für Psychiatrie und Psychotherapie. Beide Kollegen haben fundierte Kenntnisse des jeweils anderen Faches. Die Behandlung findet in einfachen Verhältnissen statt. Die Sozialwerke Pfarrer Sieber werden durch Spenden finanziert.

\section{Fallbeispiel}

Herr S. war zur Zeit des Eintritts in die Klinik SuneEgge 44 Jahre alt und blieb ein halbes Jahr in stationärer Behandlung. Er wurde von einer Heroinabgabestelle zur Behandlung der multiplen chronischen Ulzera an beiden Unterschenkeln, einer klinisch manifesten chronischen Anämie sowie zur Klärung der sozialen Situation zugewiesen. Der Patient hatte seine Wunden seit 8 Jahren (!) niemandem gezeigt und sie selber mit Taschentüchern und Papierservietten verbunden. Der Patient zeigte eine deutliche Abwehr gegen öffentliche Einrichtungen, wie zum Beispiel Ämter und Spitäler.

Eintrittsdiagnosen: Multiples, grossflächiges, chronisches Ulkus an den Unterschenkeln beidseits, Polytoxikomanie (Heroin, Kokain, Benzodiazepine) seit Jahren, rezidivierende Anämie, Eisenmangel, chronischer Blutverlust bei Ulzera und intravenöser Drogenabhängigkeit (IVDA), chronische Mangelernährung im Rahmen von Verwahrlosung, narzisstische Persönlichkeitsstörung, rezidivierende depressive Störung, gegenwärtig remittiert.
Krankheitsanamnese: Beckenvenenthrombose 1998, Tiefe Beinvenenthrombosen 1994 und 1995, Status nach Bakteriämie, Endokarditis, Pneumonie, Hepatitis B und A, Status nach Hepatitis C, PCR aktuell negativ.

Verlauf: Nach Abnahme des selbstgebastelten Verbandes imponierten auf beiden Unterschenkeln multiple Ulzera (Abb. 1). Die Analyse der Wundabstriche ergab ein reichliches Wachstum von Staphylococcus aureus und Pseudomonas aeruginosa. In einer ersten Phase wurden die Ulzera gesäubert, desinfiziert und mit Iodverbänden behandelt. Anschliessend wurde mit Feuchtverbänden (Tenderwet $^{\circ}$ ) die Granulation gefördert. Abschliessend mit Salbengaze verbunden. Der Verlauf der Wundheilung wurde fotographisch dokumentiert (Abb. 1). Initial wurde zusätzlich eine Antibiose über 4 Wochen mit Co-Amoxicillin und Ciprofloxacin peroral durchgeführt. Bei dem sehr ängstlichen und misstrauischen Patienten war eine Blutentnahme seit Monaten anstehend. Es gelang uns erstmals zwei Wochen nach Eintritt, den Patienten für eine Kontrolle der Blutwerte zu motivieren. Es konnte eine ausgeprägte Anämie (Eintritt 6,4 g\% - Austritt 13,2 g\%) festgestellt werden, die wir als Folge des jahrelangen intravenösen Drogenkonsums (IVDA) und einer mangelnden Ernährung betrachten. Der Patient erhielt im Sune-Egge eine hochwertige und gesunde Ernährung sowie eine Eisen- und Vitaminsubstitution.

Die soziale und finanzielle Situation des Patienten war bei Eintritt in Schieflage. In Zusammenarbeit mit der Behörde konnte zwecks Revision des vorliegenden Schuldenbergs eine freiwillige Beistandschaft errichtet und eine begleitete Wohnsituation

\section{Abbildung 1}

Entwicklung der Wundverhältnisse bei Herrn S. vom Eintritt bis zum Austritt.
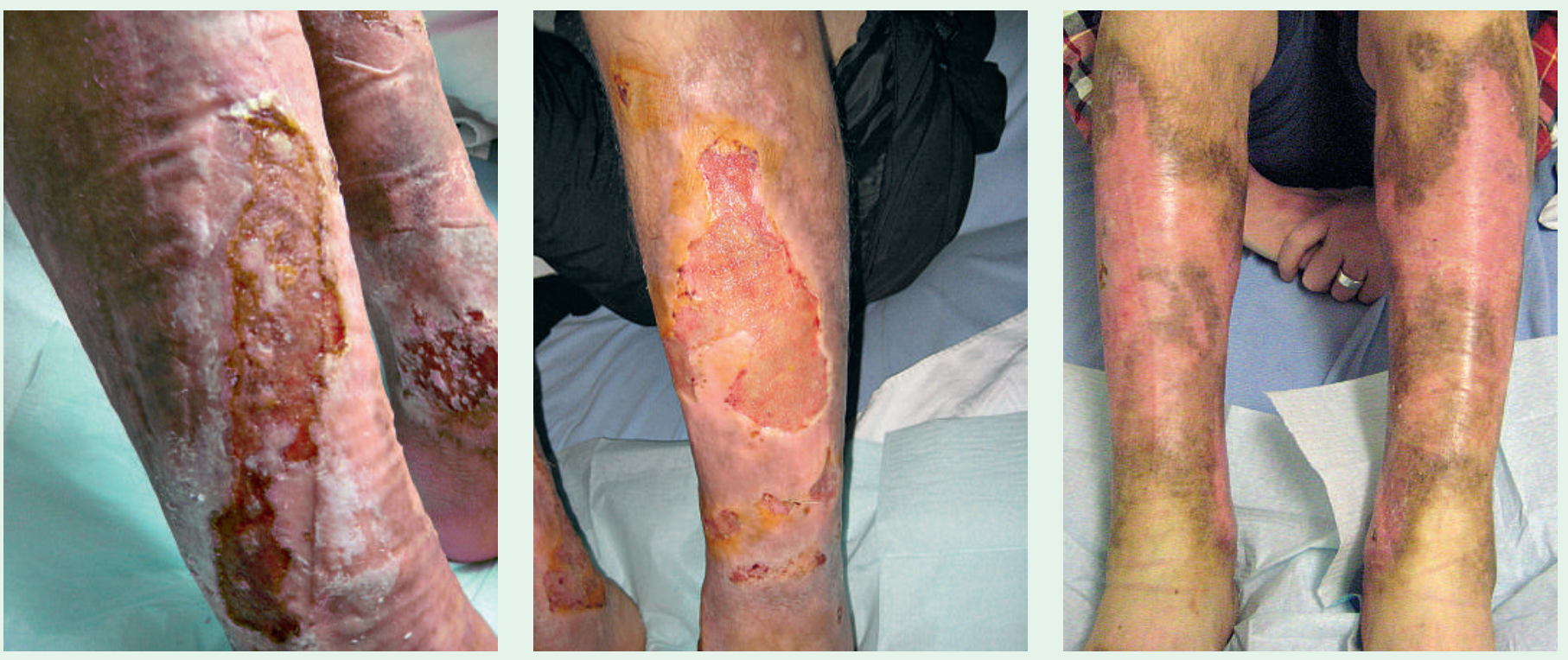
organisiert werden. Der Patient wurde nach Wundheilung und mit normalem Blutbild in geordnete Verhältnisse entlassen.

\section{Die Patientinnen und Patienten und ihre Erkrankungen}

\section{Daten}

Um einen Überblick über die behandelten Patienten und ihre Erkrankungen zu erhalten, haben wir die Daten aller 109 Patienten, 35 Frauen (32,1\%) und 74 Männer (69,9\%), die 2008 in den Sune-Egge eingetreten sind, in eine Datenbank aufgenommen, die Daten anonymisiert und ausgewertet.

Ergebnisse: Männer waren mit einem Durchschnitt von 41,1 Jahren signifikant älter als Frauen mit einem Durchschnittsalter von 37,4 Jahren (T-

Abbildung 2

Anteil der verschiedenen diagnostizierten somatischen Erkrankungen an der Gesamtzahl der Diagnosen $(n=195)$ im Jahr 2008.

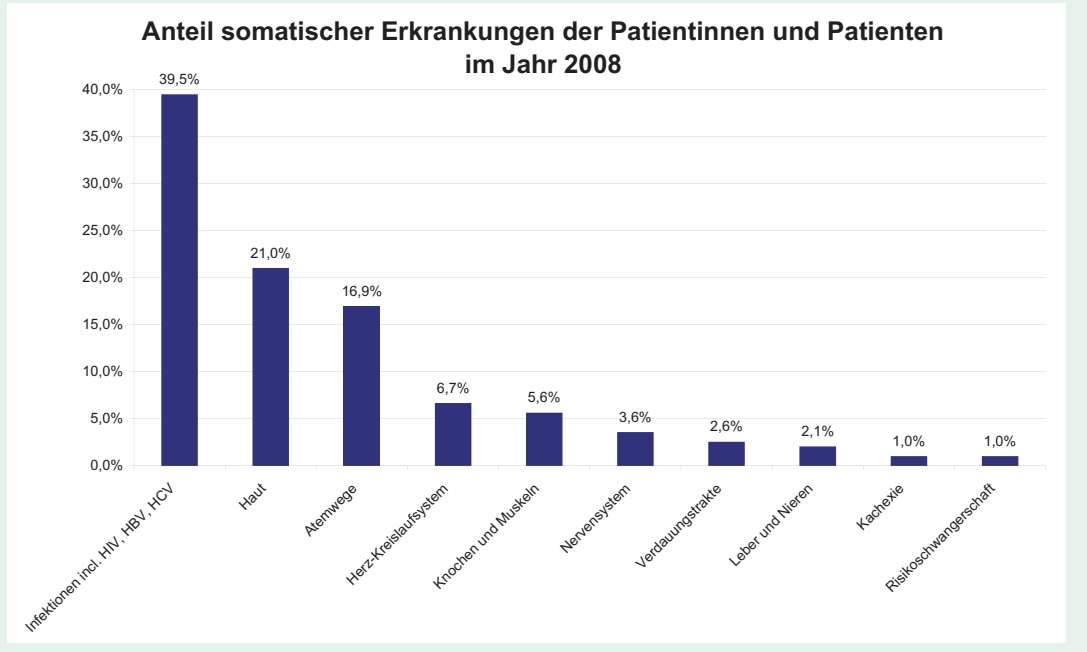

Abbildung 3

Anteil der verschiedenen diagnostizierten psychiatrischen Erkrankungen an der Gesamtzahl der Diagnosen $(n=289)$ im Jahr 2008.

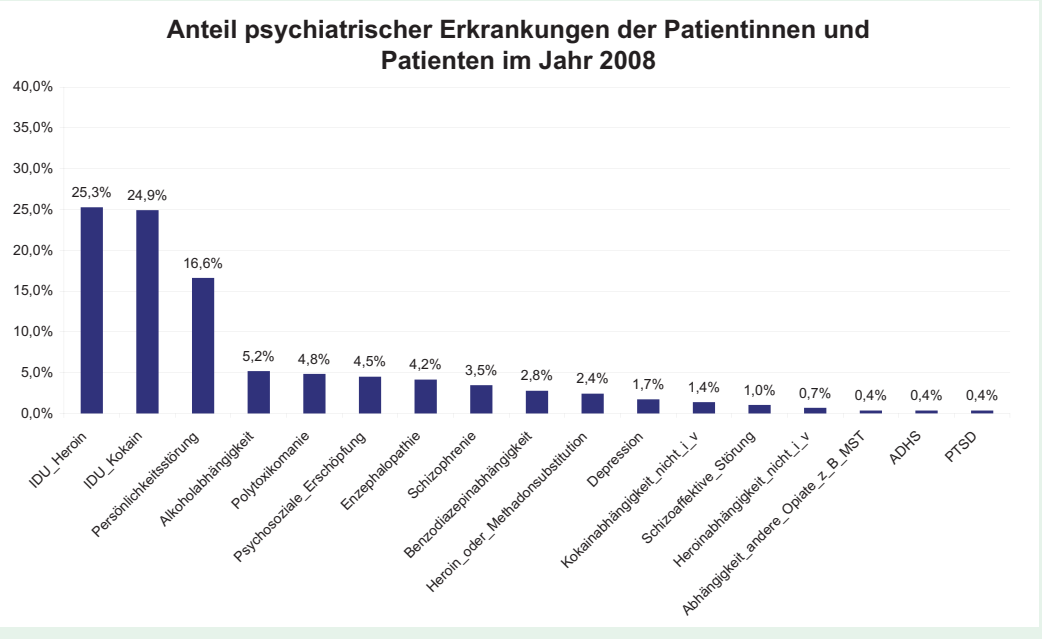

Test: $p=0,014)$. Männer blieben mit 38,1 Tagen im Durchschnitt weniger lang in der Akutbehandlung des Sune-Egge als Frauen mit 58 Tagen $(\mathrm{p}=0,0096)$. Bei der Langzeitbehandlung zeigte sich kein signifikanter Unterschied in der Aufenthaltsdauer zwischen Frauen und Männern. Alle Patienten litten unter mehreren somatischen und psychiatrischen Erkrankungen gleichzeitig.

Die somatischen Erkrankungen der Patienten stammen vorwiegend aus dem internistischen und allgemeinmedizinischen Fachgebiet. Aber auch dermatologische und neurologische Diagnosen werden nicht selten gestellt (Abb. 2). Die psychiatrischen Diagnosen gehören vorwiegend zu den Abhängigkeitserkrankungen und Persönlichkeitsstörungen. Auch affektive Störungen und Schizophrenien wurden häufig diagnostiziert (Abb. 3). Beim Blick auf die Nachbehandlungen der Patienten nach dem stationären Aufenthalt fällt auf, dass deutlich mehr Männer (13) als Frauen (2) in begleitete Wohninstitutionen eintreten. Frauen, die im Durchschnitt auch jünger sind, scheinen öfter bereit zu sein, eine psychiatrische Anschlussbehandlung zu beginnen. 53 Prozent der Patienten (55/103) mit somatischen Erkrankungen, die insgesamt 97 Prozent der Patienten ausmachen, leiden unter zwei bis vier somatischen behandlungsbedürftigen Erkrankungen gleichzeitig. Alle Patienten leiden unter psychischen Erkrankungen. 84 Prozent (90/106) davon leiden unter zwei bis fünf behandlungsbedürftigen psychischen Erkrankungen gleichzeitig.

\section{Fazit und Ausblick}

Innerhalb der nächsten 15 Jahre wird sich an der Anzahl Drogenabhängiger kaum etwas ändern. Das Durchschnittsalter wird hingegen zunehmen, nicht zuletzt wegen der gestiegenen Lebenserwartung von Menschen mit HIV-Infektionen; eine Geriatrisierung des Angebotes auch in Richtung von stationären Langzeitbehandlungen wird unausweichlich sein. Sowohl in der Suchtmedizin, in der Geriatrie und der Gerontopsychiatrie wird deshalb die Nachfrage nach interdisziplinären psychiatrisch-allgemeinmedizinischen Behandlungen steigen, wobei die Suchtpatienten in der Gruppe der älteren Patienten eher die Jüngeren ausmachen und die interdisziplinären Behandlungen in der Gruppe der Hochbetagten von der Gruppe der demenzkranken und depressiven Patienten geprägt wird. Der Sune-Egge als Modell für eine solche Institution hat sich bewährt und sollte wegen der dauerhaften Überbelegung und der vielen Abweisungen im Akutbereich vergrössert werden. Sinnvoll wäre wegen der Konzentration der Behandlungskompetenz und zur Gewährleistung der Anschlussbehandlungen, die wegen der fehlenden Integrationsmöglichkeit der Klientel in reguläre Institutionen der Langzeitpflege nicht möglich ist, ein geographisch naher Ausbau des Langzeitbereiches. 


\section{Literatur}

1 Klingemann H. National treatment systems in global perspective. Eur Addict Res. 1999;(5):109-17.

2 Baker SL. U.S. Army Heroin Abuse Identification Program in Vietnam: Implications for a Methadone Program. AJPH. 1972;(62):857-60.

3 Grob PJ. Zürcher «Needle-Park» - Ein Stück Drogengeschichte und -politik 1968-2008. Zürich: Chronos; 2009.

4 Metzger DS, Navaline H, Woody GE. Drug Abuse Treatment as AIDS Prevention. Public Health Reports. 1998;113.

5 Ron A, Rogers DE. AIDS in New York City. The Role of Intravenous Drug Users. Bull. N.Y. Acad. Med. 1989;65:787-800.

6 D`Aquila RT, Williams AB. Epidemic Human Immunodeficiency Virus (HIV) Infection Among Intravenous Drug Users (IVDU). The Yale Journal of Biology and Medicine.1987;60:545-567.

7 Selwyn PA, Hartel D, Wasserman W, Drucker E. Impact of the AIDS epidemic on morbidity and mortality among intravenous drug users in a New York City methadone maintenance program. Am J Public Health. 1989;79:1358-62.
8 Perneger TV, Giner F, Rio MD, Mino A. Randomised trial of heroin maintenance programme for addicts who fail in conventional drug treatments. BMJ. 1998;317.

9 Farrell M, Hall W. The Swiss heroin trials: testing alternative approaches - Prescribed heroin is likely to have a limited role. BMJ. 1998;316.

10 Michels II, Stöver H, Gerlach R. Substitution treatment for opioid addicts in Germany. Harm Reduct J. 2007;4:5.

11 Van den Brink W et al. Medical prescription of heroin to treatment resistant heroin addicts: two randomised controlled trials. BMJ. 2003;327.

12 Greenwood J. Creating a new drug service in Edinburgh. BrMedJ. 1990;300:587-9.

13 Michels II, Stöver H, Gerlach R. Review: Open Access Substitution treatment for opioid addicts in Germany. Harm Reduction Journal. 2007; 4. 